\title{
Antifungal Susceptibility Testing of Dermatophytes by ABDD and E-Test, a Comparative Study
}

\author{
Matheus Heita Namidi1 $^{*}$, Tejashree Ananthnaraja², Badveti Satyasai² \\ ${ }^{1}$ Applied Microbiology, Parasitology and Mycology, Oshakati Campus University of Namibia, Oshakati, Namibia \\ ${ }^{3}$ Department of Microbiology, J.S.S Medical College and Hospital, Mysuru, India \\ Email: ^mnamidi@unam.na,dr_tejashree@yahoo.co.in, badvetisatya@gmail.com
}

How to cite this paper: Namidi, M.H., Ananthnaraja, T. and Satyasai, B. (2021) Antifungal Susceptibility Testing of Dermatophytes by ABDD and E-Test, a Comparative Study. Open Journal of Medical Microbiology, 11, 129-143.

https://doi.org/10.4236/ojmm.2021.113011

Received: March 9, 2021

Accepted: August 3, 2021

Published: August 6, 2021

Copyright $\odot 2021$ by author(s) and Scientific Research Publishing Inc. This work is licensed under the Creative Commons Attribution International License (CC BY 4.0).

http://creativecommons.org/licenses/by/4.0/

\begin{abstract}
Aim: The objective of this study was to isolate, identify, and explore the in-vitro antifungal susceptibility pattern of dermatophytes isolated from clinically suspected cases of dermatophytosis (tinea infections) attending the Dermatology Department at J.S.S Hospital. Methods: This study was conducted at JSS Medical College and Hospital from December 2016 to December 2017. Clinical samples (e.g., skin scrapings and hair stumps) were collected under aseptic precautions. The identification of dermatophytes was performed through microscopic examination using $10 \%, 20 \% \& 40 \%$ potassium hydroxide $(\mathrm{KOH})$ and culture on Sabouraud dextrose agar (SDA), SDAac, PDA and Dermatophyte test medium (DTM). All dermatophytes isolates were subjected to antifungal susceptibility testing using the agar-based disk diffusion (ABDD) and E-test method against Terbinafine, Itraconazole, Fluconazole, and Griseofulvin. Data were analyzed by using Chi square test. Results: A 100 samples were studied, $46 \%$ tinea corporis, $2 \%$ tinea cruris, $9 \%$ tinea pedis, $5 \%$ tinea faciei. The dermatophytes isolated were Trichophyton rubrum 11 (35\%), Trichophyton mentagrophyte 8 (25\%), Trichophyton tonsurans 5 (16\%), Microsporum gypseum 3 (10\%), Trichophyton verrucosum 2 (6\%), Trichophyton violaceum 1 (3\%) and Microsporum audouinii 1 (3\%). Out of 31 dermatophytes 17 were sensitive to all four antifungal agents within the range of FLC $(2-6 \mathrm{mcg} / \mathrm{ml})$, ITR $(0.125-2)$, TER $(0.125-2)$, and GRI $(0.125-2), 5$ isolates were resistant in which 2 were resistant to FLC $(64.256 \mathrm{mcg} / \mathrm{ml}), 2$ isolates were resistant to TER $2(32.38 \mathrm{mcg} / \mathrm{ml}), 1$ isolate was resistant to both GRI and TER $(16.32 \mathrm{mcg} / \mathrm{ml})$ and 9 isolates were within the intermediate range. Conclusion: Every patient with a tinea infection should be properly studied for a mycological examination and should be treated accordingly. Dermatophyte test medium is more useful as an identification medium in the
\end{abstract}


isolation of dermatophytes. The ABDD method appears to be a simple, costeffective, and promising method for the evaluation of antifungal susceptibility of dermatophytes. E-test method is the most sensitive method due to the fact that quantitative MICs can be obtained directly from the E-strip. However, the E-test method is expensive and difficult in defining the precise borders of the inhibition zones in dermatophytes.

\section{Keywords}

Dermatophytosis, Dermatophyte Test Medium, Disc Diffusion and E-Strips, Antifungal Agents

\section{Introduction}

Dermatophytes are a group of closely related filamentous fungi able to damage and utilize keratin found in the skin, hair and nails [1] [2]. These are classified into three genera: Microsporum, Trichophyton, and Epidermatophyton. Dermatophytoses is an infection produced by dermatophytic fungi in the keratinized tissues. Clinically, dermatophytoses can be classified depending on the site involved. These include Tinea capitis (scalp), Tinea corporis (non-hairy skin of the body), Tinea ungium (nail infection), tinea cruris (groin), Tinea pedis (athletes foot), and Tinea barabae (bearded areas of the face and neck) [1] [2]. World Health Organization (WHO) estimates dermatophytes affect about $25 \%$ of the world's population. It is also estimated that $30 \%-70 \%$ of adults are asymptomatic carriers of these pathogens, and that the incidence of this disease increases with age [1] [3]. The estimated life-time risk of acquiring dermatophytoses is between $10 \%-20 \%$. The global prevalence of dermatophytoses is estimated to be $20 \%$ [2] [4]. The prevalence of dermatophytes has increased tremendously in the last few decades due to various factors like climatic changes, socio-economical and occupational situations [5] [6].

The diagnosis of a dermatophytic infection is mostly done clinically, but often confused with other skin infections due to the topical application of steroid ointments and creams, leading to further misdiagnosis and mismanagement [4] [7]. Hence, there arises the need for the correct, efficient, and rapid laboratory diagnosis of dermatophytes [8]. Another important point to consider is that resistance to antifungals has started appearing in dermatophytes [9]. The establishment of a reference antifungal susceptibility testing method may allow the clinician to select the appropriate therapy for the treatment of infections caused by dermatophytic fungi [10] [11].

The antifungal susceptibility test of dermatophytes has been well-studied in some developed countries, but few data are available in tropical countries [12]. Resistance to antifungal agents is also on the increase in dermatophytes seeks to improve knowledge of the molecular identification and the antifungal susceptibility test of dermatophytes [13]. The agar-based disk diffusion (ABDD) suscep- 
tibility method for dermatophytes is quick, easy, and inexpensive and does not require specialized equipment, making it a good option. The E-test results correlate well with those of the disk diffusion method [14] [15]. Unlike disk diffusion, E-test MICs are unaffected by drug properties such as molecular weight, aqueous solubility as well as diffusion characteristics or by varying growth rates of different fungi [8]. In this study, we will adopt the agar based diffusion (ABDD) method and the E-test method to assess the occurrence of resistance in Trichophyton species against Fluconazole, Itraconazole, Terbinafine and Griseofulvin. The criteria for IZDs as to whether a particular isolate is resistant, intermediate or sensitive will be based on a criteria used by R. K. Agarwal et al. (2015) in their article on Antifungal Susceptibility Testing of Dermatophytes by Agar Based Disk Diffusion Method.

\section{Materials and Methods}

This study was carried out in the Department of Medical Microbiology and Department of Dermatology in the J.S.S Medical College and Hospital, Mysore, between December 2017 and December 2018. All clinically suspected cases of dermatophytosis attending to the Dermatology department became part of the sample provided that they have given oral consent only. Samples were collected after obtaining that informed oral consent from the patients. The study was also approved by the ethical committee of JSS Medical College.

\section{Specimen collection}

Suspected lesions were cleaned with 70\% ethyl alcohol to remove any dirt and contaminating bacteria. Skin scales and crusts were collected from the erythematous, peripheral, actively growing margins of the lesions by scraping across the inflamed margin of the lesion into the apparently healthy tissue using the blunt edge of a sterile surgical blade onto clean glass slides. Hair specimens were collected by using epilating forceps to pluck along the base of the hair shaft, and scales were scraped from the surface using the blunt edge of a sterile surgical blade. The cutting of hair was avoided as the infection is usually confined to the root, very near the scalp's surface. Specimens were collected and sealed in sterile dry Petri dishes; they were labeled with the patient's name, age, sex, date of collection, and site of infection and subsequently brought to the laboratory for mycological examination. The samples were divided into two portions: one for microscopic examination and one for culture.

\section{Microscopic examination and fungal culture}

For direct microscopy, the samples collected were screened for the presence of fungal elements using a $10 \%, 20 \%$ and $40 \% \mathrm{KOH}$ mount is used. Two to three drops of the $\mathrm{KOH}$ were kept on a clean, grease-free glass slide. The sample (skin scraping or hair plucking) was placed in the $\mathrm{KOH}$ on the slide, and a clean cover slip was placed on the sample and pressed to prevent the formation of air bubbles. The sample was kept in $\mathrm{KOH}$ and then observed after $5-8$ minutes. $\mathrm{KOH}$ increases the sensitivity of the preparation and softens keratin. Each slide was 
thoroughly examined under low power (10x) and high power (40x) magnification for the presence of hyphae and/or arthroconidia. On the surface of the shaft of infected hairs, the mosaic arrangement of spores was seen (ectothrix infection) or hyphal fragments and arthroconidia was seen internally (endothrix infection). After a direct microscopic examination, irrespective of the demonstration of fungal elements, hair or skin scraping specimens were inoculated in to Sabouraud dextrose agar (SDA), Sabouraud dextrose agar with antibiotics (SDAac) base and the other in a Dermatophyte test Medium agar base both supplemented with chloramphenicol (acts as a broad spectrum antibiotic, which inhibits a wide range of gram-positive and gram-negative bacteria) and cycloheximide (to inhibit saprophytic fungi). To prepare selective media, one vial of Dermatophyte Selective Supplement DS0075 was added to $500 \mathrm{ml}$ of the medium, resulting in concentrations of $0.4 \mathrm{~g} / \mathrm{l}$ of cycloheximide and $0.05 \mathrm{~g} / \mathrm{l}$ of chloramphenicol. Cultures were incubated aerobically at room temperature $\left(25^{\circ} \mathrm{C}\right)$ for up to 4 weeks. Positive cultures were examined both macroscopically (color of the surface and reverse, topography, and texture) and microscopically (two types of conidia were formed by dermatophytes: small unicellular microconidia and larger septate macroconidia) for species identification. In the absence of any growth after 4 weeks, the culture was considered.

\section{Anti-fungal susceptibility (ABDD and E-test)}

Preparation of inoculums: The isolated fungal colonies were transferred into $5 \mathrm{ml}$ of sterile saline $(0.9 \%)$, and the suspensions were made by gently probing the surface with the tip of a sterile Pasteur pipette. Heavy particles of the suspension, when present, were allowed to settle for 15 minutes at room temperature and the upper homogenous suspension was used for further testing. The suspensions were mixed with a vortex mixer for 15 seconds and adjusted with sterile normal saline to match the opacity of 0.5 McFarland's standard.

Agar based disc diffusion method: The antifungal susceptibility testing were performed according to the National Committee for Clinical Laboratory Standards NCCLS (M38-A) guidelines. The isolated dermatophytes were sub-cultured on potato dextrose agar and incubated at $28^{\circ} \mathrm{C}$ to enhance sporulation for one week. Following growth, conidia were harvested in sterile saline and conidial suspension was adjusted to $10 \times 10^{6}$ using hemocytometer. Only two Antifungal discs were tested against the dermatophyte isolates. The discs used were Itraconazole $(10 \mathrm{mg})$ and Fluconazole $(25 \mathrm{mg})$. Muller Hinton Agar (MHA) plates were streaked evenly in three directions with a sterile cotton swab dipped into the standardized inoculums suspension. Plates were allowed to dry then antifungal discs were placed onto the medium. Each disc was pressed down to ensure complete contact with the agar surface and distributed evenly so that they are no closer than $24 \mathrm{~mm}$ from each other, center to center as illustrated by Figure 1 and Figure 2. The agar plates were then incubated at $37^{\circ} \mathrm{C}$. After 3 to 5 days of incubation, each plate was examined. The zones of inhibition were uniformly circular with a confluent lawn of growth. The diameters of the zones of complete 
inhibition were measured with an inhibition zone scale.

\section{E-test}

The E-test method was performed according to the manufacturer's protocol. The isolated dermatophytes were sub-cultured on potato dextrose agar and incubated at $28^{\circ} \mathrm{C}$ to enhance sporulation for one week. Four Antifungal agents were tested against the dermatophyte isolates. The antifungal agents were Itraconazole $(0.02-32 \mathrm{mcg} / \mathrm{ml})$, Terbinafine $(0.02-32 \mathrm{mcg} / \mathrm{ml})$, Griseofulvin (0.02 $32 \mathrm{mcg} / \mathrm{ml}$ ) and Fluconazole (0.016 - $256 \mathrm{mcg} / \mathrm{ml})$. Muller Hinton Agar (MHA) plates were streaked evenly in three directions with a sterile cotton swab dipped into the standardized inoculums suspension. Plates were allowed to dry then antifungal E-strips were placed onto the medium with a pair of forceps. The plates were incubated at $28^{\circ} \mathrm{C}$ and the results were read at 72 hours for T. mentagrophytes and 96 hours for other all species. The E-strips were aseptically removed from the

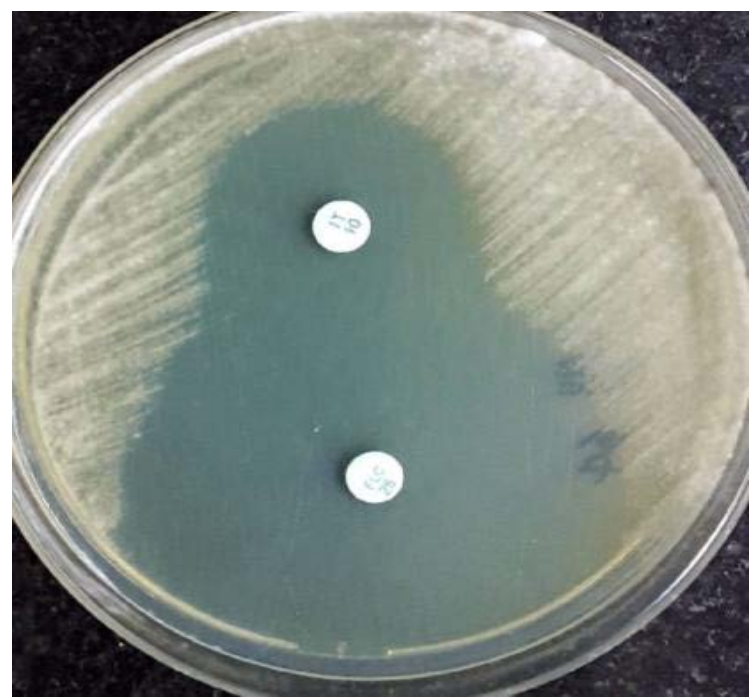

Figure 1. Sensitive T. rubrum to TRB and FLC.

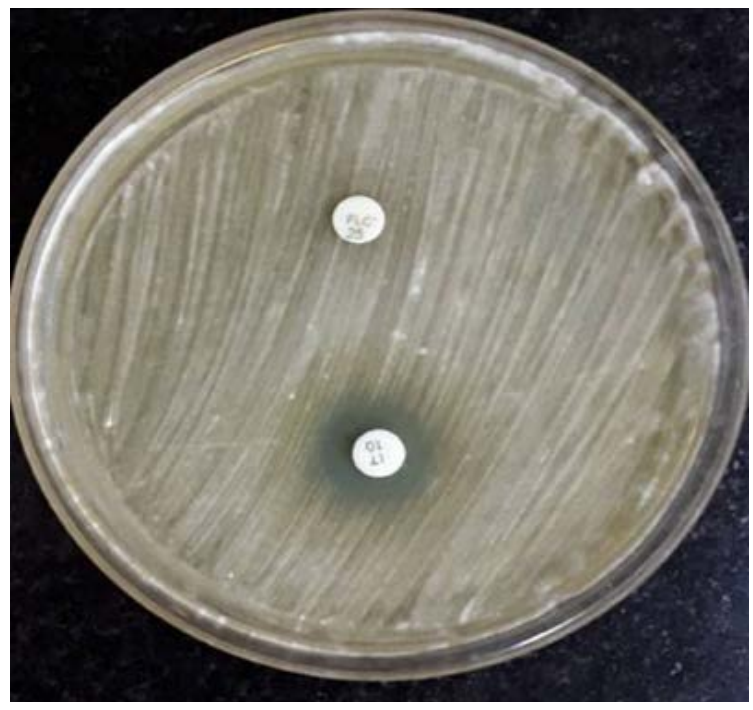

Figure 2. Resistant T. rubrum to TRB and FLC. 
packages and place on a dry clean surface of MHA plate to, and that the concentration maximum is nearest the rim of the plate. The whole length of the strip is to be in complete contact with the agar surface as illustrated by Figure 3 and Figure 4. The inhibition ellipse will form because the antifungals will diffuse across the porous paper strip. If air pockets were seen underneath the strip, they were removed by pressing gently onto the strip (without moving the strip) with a pair of forceps, moving from the minimum concentration upwards. Small bubbles under the strip would not affect the results. Once applied, the strip was not to be moved because of the instantaneous release of drug into the agar. The IZD were recorded in the results section.

\section{Determination of MIC endpoints}

In general, MIC was defined as the lowest drug concentration at which the border of the elliptical inhibition zone intercepted the MIC scale on the E-test strip. When a double halo of growth was observed, the MIC was read at the point where growth was completely inhibited. When different intersections were observed on either side of the strip, the highest MIC value was read and recorded in Table 7.

\section{Results}

In the present study, a total of 100 clinically suspected cases of nail, skin and hair dermatophytosis were included in the study. Out of the 100 samples 85 (85\%) were skin scrappings, $10(10 \%)$ were hair and 5 (5\%) were nail samples as illustrated in Table 1. Furthermore, 56 (56\%) samples were collected from male patients and $34(34 \%)$ samples were collected from female patients. The samples in this study were from patients in the age group of 31 - 40 (27\%), 51 - 60 (13\%), 41 - 50 (13\%), 11 - 20 (12\%), 1 - 10 (11\%), 21 - 30 (10\%), 61 - 70 (9\%), 71 - 80 (4\%)

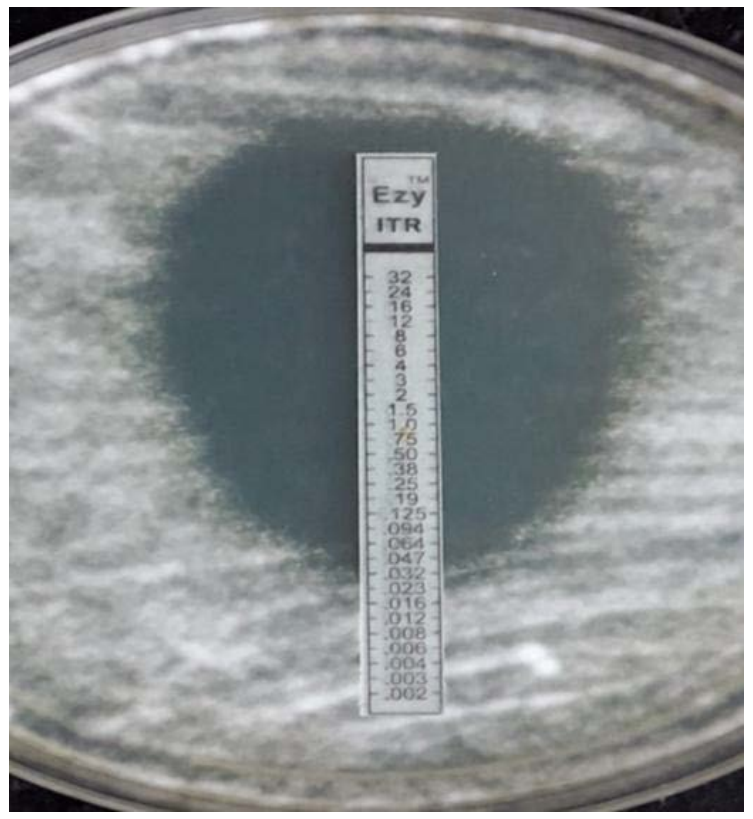

Figure 3. Sensitive T. rubrum. 
and $81-90(1 \%)$. All the samples were subjected to $\mathrm{KOH}$ microscopy and culture. Out of 100 samples 28 (28\%) of them were $\mathrm{KOH}$ positive and as well as culture positive, $3(3 \%)$ were $\mathrm{KOH}$ negative and culture positive, $\mathrm{KOH}$ positive and culture negative were 5 (5\%). However, 64 (64\%) samples were both $\mathrm{KOH}$ and culture negative as illustrated in Table 2. Table 3 illustrates that tinea corporis were the highest at $64 \%$ of the identified isolates while tinea unguium and tinea faceie made up $5 \%$ each. Table 4 shows the number of respective dermatophytes

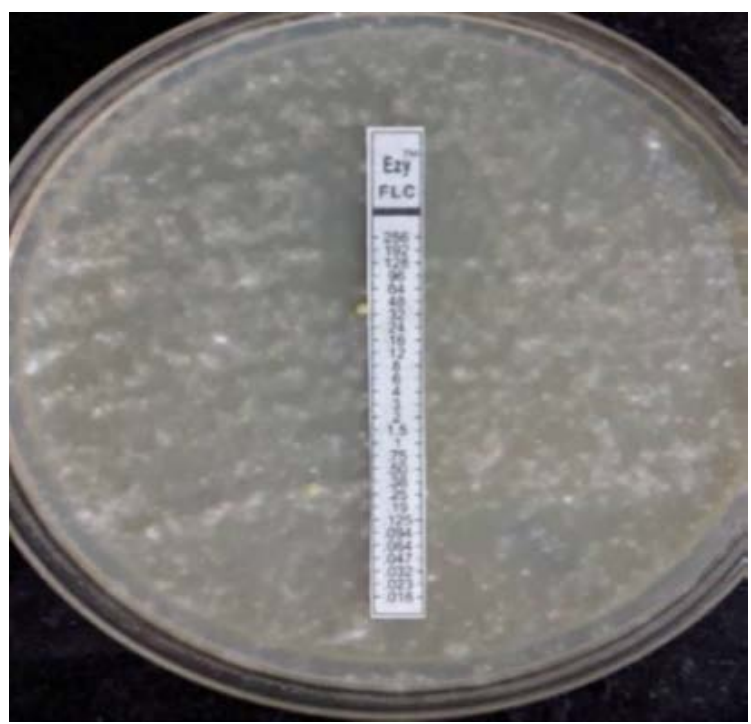

Figure 4. Resistant T. rubrum.

Table 1. Sample-wise distribution.

\begin{tabular}{cccc}
\hline Sample & Skin & Hair & Nail \\
\hline Number & 85 & 10 & 5 \\
Percentage \% & 85 & 10 & 5 \\
\hline
\end{tabular}

Table 2. Direct microscopy and culture.

\begin{tabular}{cccc}
\hline Total samples (100) & KOH positive & KOH negative & Total \\
\hline Culture positive & $28(28 \%)$ & $3(3 \%)$ & $31(31 \%)$ \\
Culture negative & $5(5 \%)$ & $64(64 \%)$ & $69(69 \%)$ \\
\hline
\end{tabular}

Table 3. Clinical type distribution of samples.

\begin{tabular}{ccc}
\hline Sample & No of samples & Percentage \% \\
\hline Tinea corporis & 64 & 64 \\
Tinea cruris & 6 & 6 \\
Tinea pedis & 10 & 10 \\
Tinea faceie & 5 & 5 \\
Tinea capitis & 10 & 10 \\
Tinea unguium & 5 & 5 \\
\hline
\end{tabular}


as identified. Table 5 was derived from R. K. Agarwal et al. (2015) and was used as the criteria for classifying the isolates' IZD as resistant, intermediate or sensitive while Table 6 shows MICs criteria as provided by Himedia. The IZD were then carefully recorded in Table 7, Table 8 and Table 9 respectively.

Table 4. Number of organisms isolated.

\begin{tabular}{ccc}
\hline Organism & Number of organisms & Percentage\% \\
\hline T. rubrum & 11 & 35 \\
T. mentagrophyte & 8 & 25 \\
T. tonsurans & 5 & 16 \\
T. verrucosum & 2 & 6 \\
M. gypsium & 3 & 10 \\
T. violacium & 1 & 3 \\
M. audoinii & 1 & 3 \\
\hline
\end{tabular}

Table 5. Criteria cut off values for IZDs for each of the four drugs ${ }^{\mathrm{a}}$.

\begin{tabular}{ccccc}
\hline & \multicolumn{3}{c}{ Inhibition zone diameters } \\
\cline { 2 - 5 } Drugs & Mean \pm SD & $\begin{array}{c}\text { Sensitive } \\
\text { Mean } \pm 1 \text { SD }\end{array}$ & $\begin{array}{c}\text { Intermediate Sensitive } \\
\text { Mean-1 SD to Mean-2 SD }\end{array}$ & $\begin{array}{c}\text { Resistant } \\
<\text { Mean -2 SD }\end{array}$ \\
\hline FLC & $22.6 \pm 4.2$ & $>19$ & $14-19$ & $<14$ \\
ITR & $27.3 \pm 6.2$ & $>21$ & $15-21$ & $<15$ \\
TER & $32.1 \pm 6.1$ & $>26$ & $20-26$ & $<20$ \\
GRI & $35.9 \pm 4.9$ & $>31$ & $26-31$ & $<26$ \\
\hline
\end{tabular}

${ }^{a}$ R. K. Agarwal et al. (2015) Int. J. Curr. Microbiol. App. Sci (2015) 4(3): 430-436. ABDD: Agar-based disk diffusion; ITR: Itraconazole; FLC: Fluconazole; IZD: Inhibition zone diffusion; S: Sensitive.

Table 6. Interpretative criteria for susceptibility categorization for MIC .

\begin{tabular}{cccc}
\hline & \multicolumn{3}{c}{ Interpretative criteria } \\
\hline Drug & $<\mathrm{S}$ & $\mathrm{S}^{-} \mathrm{DD}^{*}$ & $>\mathrm{R}$ \\
FLC & 8 & $16-32$ & 64 \\
ITR, TRB, GRI & $0.125-1$ & $02-4$ & $>8$
\end{tabular}

${ }^{a}$ Criteria provided by Himedia. ABDD: Agar based diffusion; FLC: Fluconazole; IZD: Inhibition zone diameter.

Table 7. E-test values for the ITR and FLC by average.

\begin{tabular}{ccccc}
\hline \multirow{2}{*}{ Dermatophyte } & \multicolumn{2}{c}{ Average IZD $(\mathrm{mm})$} & \multicolumn{2}{c}{ Average $\mathrm{MIC}(\mathrm{mcg} / \mathrm{ml})$} \\
\cline { 2 - 5 } & FLC & ITR & FLC & ITR \\
\hline T. rubrum & 29.5 & 20.9 & 24 & 0.28 \\
T. mentagrophyte & 36.3 & 29.23 & 1 & 0.12 \\
T.tonsurans & 32.6 & 30.4 & 2.2 & 0.142 \\
T.verrucosum & 34.5 & 26 & 0.048 & 26
\end{tabular}




\section{Continued}

\begin{tabular}{ccccc}
\hline M.gypsium & 35 & 29.3 & 0.98 & 0.36 \\
M.violacium & 40 & 28 & 1 & 0.25 \\
M.audoinii & 31 & 40 & 1.5 & 0.094 \\
Average & $\mathbf{3 4 . 1 2 8 5 7 1 4 3}$ & $\mathbf{2 9 . 1 1 8 5 7 1 4 3}$ & $\mathbf{4 . 3 8 9 7 1 4 2 8 6}$ & $\mathbf{3 . 8 9 2 2 8 5 7 1 4}$ \\
\hline
\end{tabular}

Table 8. ABDD values for the ITR and FLC by average.

\begin{tabular}{cccc}
\hline Dermatophyte & ITR & Average IZD (mm) & \\
\hline T. rubrum & 268 & 24.36363636 & $\mathrm{~S}$ \\
T. mentagrophyte & 233 & 29.125 & $\mathrm{~S}$ \\
T. tonsurans & 152 & 30.4 & $\mathrm{~S}$ \\
T. verrucosum & 52 & 26 & $\mathrm{~S}$ \\
M. gypsium & 81 & 27 & $\mathrm{~S}$ \\
M. violacium & 28 & 28 & $\mathrm{~S}$ \\
M. audoinii & 40 & 40 & $\mathrm{~S}$ \\
\hline
\end{tabular}

Table 9. ABDD values for FLC by average.

\begin{tabular}{cccc}
\hline Dermatophyte & FLC & Average IZD (mm) & \\
\hline T. rubrum & 311 & 28.3 & $\mathrm{~S}$ \\
ST. Mentagrophyte & 256 & 32 & $\mathrm{~S}$ \\
T. tonsurans & 163 & 32.6 & $\mathrm{~S}$ \\
T. verrucosum & 69 & 34.5 & $\mathrm{~S}$ \\
M. gypsium & 105 & 35 & $\mathrm{~S}$ \\
M. violacium & 40 & 40 & $\mathrm{~S}$ \\
M. audoinii & 31 & 31 & $\mathrm{~S}$
\end{tabular}

\section{Discussion}

Superficial cutaneous fungal infections are commonly encountered fungal diseases prevalent in most parts of the world [8] [16]. The dermatophytes are by far the most significant cutaneous fungi because of their widespread involvement of population at large and their worldwide prevalence [16] [17]. Dermatophytoses form over $50 \%-75 \%$ of all the mycological infections. The diagnosis of a dermatophytic infection is mostly done clinically, but often confused with other skin infections due to the topical application of steroid ointments and creams, leading to further misdiagnosis and mismanagement [18]. Hence, there arises the need for the correct, efficient, and rapid laboratory diagnosis of dermatophytes [5] [19]. Another important point to consider is that resistance to antifungals has started appearing in dermatophytes [20]. The establishment of a reference antifungal susceptibility testing method may allow the clinician to select the appropriate therapy for the treatment and also for studying mechanisms of 
drug resistance of dermatophytic fungi [21] [22].

In the present study a total of 100 specimens (skin scrapings, hair fragments and nail clippings) were collected from clinically suspected cases of dermatophytosis. 69\% were from males and 31 (31\%) were from female patients visiting the dermatology department. Out of the 100 clinical cases, tinea corporis accounted for $64 \%$, tinea cruris accounted for $6 \%$, tinea pedis $10 \%$, tinea faciei $5 \%$, tinea capitis $10 \%$ and tinea unguium accounted for $5 \%$ and. In a study done by Bindu et al. in (2016), tinea corporis accounted for 35 (23\%) of the cases, tinea pedis in $16(10 \%)$ cases, tinea unguium $5(10 \%)$ cases, tinea capitis in $10(6 \%)$ cases, tinea faciei in $5(3 \%)$ cases and least cases were tinea barbae $2(1 \%)$. A study by Anupama. A et al. (2017) had similar findings in which out of 100 cases male proportion were 73 (73\%) and 27 (27\%).

The specimens were examined by direct microscopic examinations by potassium hydroxide $(\mathrm{KOH})$ wet mount and by culture methods. Out of a 100 samples, $64 \%$ were $\mathrm{KOH}$ negative and $36 \%$ were $\mathrm{KOH}$ positive. Out of 100 samples 31 (31\%) were culture positive and $69(69 \%)$ were culture negative, 28 (90.3\%) were both culture and $\mathrm{KOH}$ positive, $3(9.6 \%)$ were $\mathrm{KOH}$ negative and culture positive and $5(16 \%)$ were $\mathrm{KOH}$ positive and culture negative. Anupama. A et al. (2017) also reported similar proportions whereby out of 100 clinical samples $58 \%$ samples were culture positive and $\mathrm{KOH}$ positive, 19\% samples were Culture positive and $\mathrm{KOH}$ negative, $18 \%$ samples were Culture as well as $\mathrm{KOH}$ negative. $5 \%$ samples were $\mathrm{KOH}$ positive and culture negative.

In culture, the isolated dermatophyte isolates were identified by macroscopic morphological characteristics (pigmentation, growth rate and texture etc.) and followed by microscopic examination. The isolated organisms were identified as Trichophyton rubrum 11 (35\%), Trichophyton mentagrophyte 8 (25\%), Trichophyton tonsurans 5 (16\%), Microsporum gypseum 3 (10\%), Trichophyton verrucosum 2 (6\%), Trichophyton violaceum 1 (3\%) and Microsporum audouinii 1 (3\%). In a study done by Keyvan Pakshir et al. in (2009), similar findings were reported but in their study Trichophyton mentagrophytes accounted for 13 (32.5\%), T. rubrum 8 (20\%), T. violaceum 4 (10\%), Microsporum gypseum 3 (7.5\%), $T$. tonsurans 2 (5\%), T. verucosum $2(5 \%)$ respectively.

The 31 isolated dermatophytes were tested for antifungal susceptibility testing by Agar based disc diffusion (ABDD) with FLC $(25 \mathrm{mcg} / \mathrm{ml})$ and ITR (10 $\mathrm{mcg} / \mathrm{ml})$. Out of 31 dermatophytes $25(80 \%)$ isolates were sensitive to both FLC and ITR, $5(16 \%)$ strains were intermediate within the range of $(15-20 \mathrm{~mm})$ for ITR and FLC (14 - $19 \mathrm{~mm})$ respectively. $1(4 \%)$ of the isolates were resistant to both ITR $(14 \mathrm{~mm})$ and FLC $(6 \mathrm{~mm})$. The 31 dermatophytes were further tested for antifungal susceptibility testing by E-test with four antifungal agents namely Fluconazole $(0.016-256 \mathrm{mcg} / \mathrm{ml})$, Itraconazole $(0.002-32 \mathrm{mcg} / \mathrm{ml})$, Terbinafine $(0.002-32 \mathrm{mcg} / \mathrm{ml})$, and Griseofulvin $(0.002-32 \mathrm{mcg} / \mathrm{ml})$. Out of 31 dermatophytes 17 were sensitive to all four antifungal agents within the range of FLC (2 $6 \mathrm{mcg} / \mathrm{ml})$, ITR $(0.125-2)$, TER $(0.125-2)$, and GRI $(0.125-2), 5$ isolates were 
resistant in which 2 were resistant to FLC $(64 ; 256 \mathrm{mcg} / \mathrm{ml}), 2$ isolates were resistant to TER (32; $38 \mathrm{mcg} / \mathrm{ml}), 1$ isolate was resistant to both GRI and TER (16; $32 \mathrm{mcg} / \mathrm{ml}$ ) and 9 isolates were within the intermediate range.

A comparison was made between the two antifungal agents used in both tests (E-test and ABDD). The MIC and IZD values produced by the organism were recorded in Table 4, Table 8 and Table 9. The MIC and the IZD values were found to be inversely proportional to each other [23]. In a study done by Agarwal et al. (2015), similar IZD values of FLU (13 - $30 \mathrm{~mm})$, ITR (20 - $35 \mathrm{~mm})$ were also been reported. In our study the highest MIC values for FLC were (32 $64 \mathrm{mcg} / \mathrm{ml}$ ). Sanjivan et al. (2015) also reported similar MIC values of FLC (34 $\mathrm{mcg} / \mathrm{ml})$. However, it is difficult to compare results of the E-test and ABDD methods due to variability in critical technical factors in different studies, including inoculums size, type of media, incubation temperature and time of reading, which may explain the different results in antifungal susceptibility testing obtained by various investigators and laboratories [24].

The zones of inhibition were seen in all the strains except 1 strains of $T$. rubrum as could be seen in Figure 1 and Figure 2. The zone of inhibition varied from $0>40 \mathrm{~mm}$ for Fluconazole. In E-test, the MIC varied from $256-0.125$ $\mathrm{mcg} / \mathrm{ml}$ for Fluconazole, 2 - $4 \mathrm{mcg} / \mathrm{ml}$ for Itraconazole, 0 - $48 \mathrm{mcg} / \mathrm{ml}$ for Terbinafine and $0-3 \mathrm{mcg} / \mathrm{ml}$ for Griseofulvin. In a study conducted by Shalini Gupta et al. (2015) similar MIC values in close ranges were reported of four antifungal agents, FLC (32 - $64 \mathrm{mcg} / \mathrm{ml}$, ITR ( $4-6 \mathrm{mcg} / \mathrm{ml})$. Although some in vitro antifungal susceptibility tests are now available including those mentioned in the CLSI document regarding filamentous fungi (CLSI, 2008, 2010, no simple reference method has been standardised for testing the drug susceptibility of dermatophytes) [25] [26].

Experience in determining MICs and careful attention to procedural details are critically important in conducting the E-test method because it is not as easy as it is with inhibition zone scale in disk diffusion method [2] [27]. The MICs and IZDs are inversely proportional to each other i.e. when the MIC for the drug is more; the IZD is smaller and vice versa. Successful treatment of fungal infections depends on the ability of a given antimycotic agent to eradicate the fungus from the tissue [28]. Though some in-vitro antifungal susceptibility tests are now available, no simple reference method has been standardized for testing the drug susceptibility of dermatophytes [28] [29].

\section{Conclusions}

The present study demonstrated a good correlation between MICs and IZDs of the drugs. It was observed that if the MIC value was low for a particular isolate a larger zone of inhibition was observed [30]. Furthermore, it was also observed that if MIC was higher, a smaller zone of inhibition was observed.

Disc diffusion method is simple, reproducible, cheap and easily adaptable. Furthermore, it has potential for use in selection of appropriate antifungal agents 
once the conditions such as temperature and inoculums size are properly standardized [31] [32]. The inhibition zones for the disks were easy to measure with the inhibition zone scale compared to the reading of the E-strips. In addition to that, more than one antifungal can be tested in the same plate at the same time to conserve time and media [18] [33]. Antifungal susceptibility testing by disk diffusion can become an important method in treatment of patients with fungal infections.

Therefore, based on the results of the present study; it could be concluded that the method for antifungal susceptibility of dermatophytes is the disc diffusion method as is the simpler, cheap and reliable method in comparison with the E-test. However, when it comes to sensitivity the E-test supersedes the disc diffusion method. Previous studies have also shown that the E-test method is the most sensitive method due to the fact that quantitative MICs can be obtained directly from the E-strip [34] [35]. However, the E-test method is expensive and difficult in defining the precise borders of the inhibition zones in dermatophytes [36]. Despite that, the agar based disk diffusion method was found to be favorable in comparison to the E-test method. Future efforts must put more emphasis on establishing standard interpretive break-points for dermatophytes for licensed as well as newly introduced antifungals and correlating them to the clinical outcomes.

\section{Conflicts of Interest}

The authors declare no conflicts of interest regarding the publication of this paper.

\section{References}

[1] Ghannoum, M.A., Chand, D.V., et al. (2006) Susceptibility Testing of Dermatophytes.

[2] Shalaby, M.M., Nasr-El-din, A. and Abu El-Hamd, M. (2016) Isolation, Identification, and in Vitro Antifungal Susceptibility Testing of Dermatophytes from Clinical Samples at Sohag University Hospital in Egypt. Electronic Physician, 8, 2557-2567. https://doi.org/10.19082/2557

[3] Sagar, K. and Vidyasagar, G.M. (2013) Antidermatophytic Activity of Some Traditionally Used Medicinal Plants of North Karnataka Region. Journal of Applied Pharmaceutical Science, 3, 77-83.

[4] Agarwal, R.K., Gupta, S., Mittal, G., Khan, F., Roy, S. and Argawal, A. (2015) Antifungal Susceptibility Testing of Dermatophytes by Agar Diffusion Method. International Journal of Current Microbiology and Applied Sciences, 4, 430-436.

[5] Mercy, K.A., Ijeoma, I. and Emmanuel, K.J. (2014) Anti-Dermatophytic Activity of Garlic (Allium sativum) Extracts on Some Dermatophytic Fungi. International Letters of Natural Science, 19, 34-40. https://doi.org/10.18052/www.scipress.com/ILNS.24.34

[6] Mehdi, R.M., Masoomeh, R. and Mahendra, R. (2015) Medical Mycology. Taylor \& Francis Group. https://www.taylorfrancis.com/books/edit/10.1201/b18707/

[7] Nasimuddin, S., Appalaraju, B., Surendran, P. and Srinivas, C.R. (2014) Isolation, 
Identification and Comparative Analysis of SDA and DTM for Dermatophytes from Clinical Samples in a Tertiary Care Hospital. IOSR Journal of Dental and Medical Sciences, 13, 68-73. https://doi.org/10.9790/0853-131126873

[8] Sabtharishi, V., Katragadda, R. and Ravinder, T. (2017) A Study on the Antifungal Susceptibility Pattern of Dermatophytes Isolated in a Tertiary Care Hospital. International Journal of Bioassays, 6, 5379. https://doi.org/10.21746/ijbio.2017.05.003

[9] Matnani, G., Roy, I., Gadham, N., Mandal, L.A., Ujagare, M. and Jadhav, S.V. (2012) Identification and Antifungal Susceptibility Testing of Fungal Infections in Clinical Samples of Suspected Superficial Fungal Infections. International Journal of Medical and Clinical Research, 3, 215-220.

https://doi.org/10.9735/0976-5530.3.7.215-220

[10] Sowmya, N., Appalaraju, B., Srinivas, C.R. and Surendran, P. (2015) Antifungal Susceptibility Testing for Dermatophytes Isolated from Clinical Samples by Broth Dilution Method in a Tertiary Care Hospital. JMR, 1, 64-67.

[11] Graser, Y., Scott, J. and Summerbell, R. (2007) The New Species Concept in Dermatophytes-A Polyphasic Approach. Springer Science + Business Media, Berlin.

[12] Granade, T.C. and Artis, W.M. (1980) Antimycotic Susceptibility Testing of Dermatophytes in Microcultures with a Standardized Fragmented Mycelial Inoculum. Antimicrobial Agents and Chemotherapy, 17, 725-729. https://doi.org/10.1128/AAC.17.4.725

[13] Bhargava, A. and Ahluwalia, A. (2014) Comparison of the Minimum Fungicidal Concentration of Clotrimazole, Ketoco-Nazole, Miconazole and Terbinafine against Clinical Isolates of Dermatophytes. Indian Medical Gazzete. https://www.scribd.com/document/275308707/

[14] Ebrahim, H.M., Asaad, A.M. and Amer, A. (2010) Antifungal Susceptibility Patterns of Dermatophytes Clinical Isolates from Dermatophytosis Patients before and after Therapy. Egyptian Journal of Medical Microbiology, 19, 41-46.

[15] Nigam, P.K. (2015) Antifungal Drugs and Resistance: Current Concepts. Our Dermatology Online, 6, 212-221. https://doi.org/10.7241/ourd.20152.58

[16] Fothergill, A.W., et al. (2006) Antifungal Susceptibility Testing. Infectious Disease Clinics of North America, 20, 699-709. https://doi.org/10.1016/j.idc.2006.06.008

[17] Brito, G.N.B., et al. (2011) In Vitro Antifungal Susceptibility of Candida spp. Oral Isolates from HIV-Positive Patients and Control Individuals. Brazilian Oral Research, 25, 28-33. https://doi.org/10.1590/S1806-83242011005000001

[18] Panda, S. and Verma, S. (2017) The menace of Dermatophytosis in India: The Evidence That We Need. Indian Journal of Dermatology, Venereology and Leprology, 83, 281-284. https://doi.org/10.4103/ijdvl.IJDVL_224_17

[19] Bhatia, V.K. and Sharma, P.C. (2014) Epidemiological Studies on Dermatophytosis in Human Patients in Himachal Pradesh, India. Springerplus, 3, 134. https://doi.org/10.1186/2193-1801-3-134

[20] Ramaraj, V., Vijayaraman, R.S., Rangarajan, S. and Kindo, A.J. (2016) Incidence and Prevalence of Dermatophytosis in and around Chennai, Tamilnadu, India. International Journal of Research in Medical Sciences, 4, 695-700. https://doi.org/10.18203/2320-6012.ijrms20160483

[21] Teklebirhan, G. and Bitew, A. (2015) Prevalence of Dermatophytic Infection and the Spectrum of Dermatophytes in Patients Attending a Tertiary Hospital in Addis Ababa, Ethiopia. International Journal of Microbiology, 2015, Article ID: 653419. https://doi.org/10.1155/2015/653419 
[22] Khan, S., Singhal, S., Mathur, T., Upadhyay, D.J. and Rattan, A. (2006) Antifungal Susceptibility Testing Method from Resources Constrained Laboratories. Indian Journal of Medical Microbiology, 24, 171-6.

[23] Adimi, P., Hashemi, S.J., Mahmoudi, M., Mirhendi, H., Shidfar, M.R., Emmami, M., Rezaei-Matehkolaei, A., Gramishoar, M. and Kordbacheh, P. (2013) In-Vitro Activity of 10 Antifungal Agents against 320 Dermatophyte Strains Using Microdilution Method in Tehran. Iranian Journal of Pharmaceutical Research, 12, 537-545.

[24] Indira, G. (2014) In vitro Antifungal Susceptibility Testing of 5 Antifungal Agents against Dermatophytic Species by CLSI (M38-A) Micro Dilution Method. Clinical Microbiology, 3, 145. https://doi.org/10.4172/2327-5073.1000145

[25] Tamura, T., Asahara, M., Yamamoto, M., Yamaura, M., et al. (2014) In Vitro Susceptibility of Dermatomycoses Agents to Six Antifungal Drugs and Evaluation by Fractional Inhibitory Concentration Index of Combined Effects of Amorolfine and Itraconazole in Dermatophytes. Microbiology and Immunology, 58, 1-8. https://doi.org/10.1111/1348-0421.12109

[26] Mitruka, B., et al. (2016) Study of Hospital Based Epidemiology \& Clinical Types of Cases of Dermatophytosis Presenting in Outpatient Department of Skin and Venereology. Scholars Journal of Applied Medical Sciences, 4, 1603-1616.

[27] Anupama, A. (2017) Isolation and Identification of Dermatophytes from Clinical Samples-One Year Study. International Journal of Current Microbiology and Applied Sciences, 6, 1276-1281. https://doi.org/10.20546/ijcmas.2017.611.152

[28] Mota, C.R.A., et al. (2009) Comparison of in Vitro Activity of Five Antifungal Agents against Dermatophytes, Using the Agar Dilution and Broth Microdilution Methods. Revista da Sociedade Brasileira de Medicina Tropical, 42, 250-254. https://doi.org/10.1590/S0037-86822009000300003

[29] Manoharan, A., Pai, R., Shankar, V., Thomas, K. and Lalitha, M.K. (2003) Comparison of Disc Diffusion \& E Test Methods with Agar Dilution for Antimicrobial Susceptibility Testing of Haemophilus influenzae. Indian Journal of Medical Research, 117, 81-87.

[30] Pakshir, K., Bahaedinie, L., Rezaei, Z., Sodaifi, M. and Zomorodian, K. (2009) In Vitro Activity of Six Antifungal Drugs against Clinically Important Dermatophytes. Jundishapur Journal of Microbiology, 2, 158-163.

[31] Sanjivan, L., et al. (2015) Evaluation of E Test Strips ESYMIC of Fluconazole \& HICOMB MIC of Ketoconazole for Antifungal Susceptibility Testing of Trichophyton Species.

[32] Jaruvongvanich, V., Worasilchai, N., Kongnatthasate, K., Jaruvongvanich, S., Damkerngsuntorn, W., Atikarnbodee, D., Thammahong, A., Lerdlitruangsine, S. and Chindamporn, A. (2016) Correlation between Broth Microdilution, E-Test and Disk Diffusion Methods for Testing Antifungal Susceptibility of Candida Species Isolated from Thai Blood Samples. Asian Biomedicine, 10, 75-80.

[33] Pfaller, M.A., Messer, S.A., Mills, K. and Bolmstrom, A. (2000) In Vitro Susceptibility Testing of Filamentous Fungi: Comparison of E-Test and Reference Microdilution Methods for Determining Itraconazole MICs. Journal of Clinical Microbiology, 38, 3359-3361. https://doi.org/10.1128/JCM.38.9.3359-3361.2000

[34] Espinel-Ingroff, A. (2000) Clinical Utility of in Vitro Antifungal Susceptibility Testing. Revista Española de Quimioterapia, 13, 161-166.

[35] Vandenbossche, I., Vaneechoutte, M., Vandevenne, M., De Baere, T. and Verchragen, G. (2002) Susceptibility Testing of Fluconazole by the NCCLS Broth Macrodilution Method, E-Test, and Disk Diffusion for Application in Routine Laboratory. 
Journal of Clinical Microbiology, 40, 918-921.

https://doi.org/10.1128/JCM.40.3.918-921.2002

[36] Shalini, G., et al. (2015) Comparison of Broth Micro Dilution and Disk Diffusion Method for Susceptibility Testing of Dermatophytes. International Journal of Current Microbiology and Applied Sciences, 4, 24-33. 